\title{
Bootstrap Percolation and Diffusion in Random Graphs with Given Vertex Degrees
}

\author{
Hamed Amini \\ École Normale Supérieure - INRIA Rocquencourt, Paris, France \\ hamed.amini@ens.fr
}

Submitted: Jul 31, 2009; Accepted: Jan 26, 2010; Published: Feb 8, 2010

Mathematics Subject Classifications: 05C80

\begin{abstract}
We consider diffusion in random graphs with given vertex degrees. Our diffusion model can be viewed as a variant of a cellular automaton growth process: assume that each node can be in one of the two possible states, inactive or active. The parameters of the model are two given functions $\theta: \mathbb{N} \rightarrow \mathbb{N}$ and $\alpha: \mathbb{N} \rightarrow[0,1]$. At the beginning of the process, each node $v$ of degree $d_{v}$ becomes active with probability $\alpha\left(d_{v}\right)$ independently of the other vertices. Presence of the active vertices triggers a percolation process: if a node $v$ is active, it remains active forever. And if it is inactive, it will become active when at least $\theta\left(d_{v}\right)$ of its neighbors are active. In the case where $\alpha(d)=\alpha$ and $\theta(d)=\theta$, for each $d \in \mathbb{N}$, our diffusion model is equivalent to what is called bootstrap percolation. The main result of this paper is a theorem which enables us to find the final proportion of the active vertices in the asymptotic case, i.e., when $n \rightarrow \infty$. This is done via analysis of the process on the multigraph counterpart of the graph model.
\end{abstract}

\section{Introduction}

The diffusion model we consider in this paper is a generalization of bootstrap percolation in an arbitrary graph (modeling a given network). Let $G=(V, E)$ be a connected graph. Given two vertices $i$ and $j$, we write $i \sim j$ if $\{i, j\} \in E$. The threshold associated to a node $i$ is $\theta\left(d_{i}\right)$ where $d_{i}$ is the degree of $i$ and $\theta: \mathbb{N} \rightarrow \mathbb{N}$ is given fixed function. Assume that each node can be in one of the two possible states: inactive or active. Let $\alpha: \mathbb{N} \rightarrow[0,1]$ be a fixed given function. At time 0 , each node $i$ becomes active with probability $\alpha\left(d_{i}\right)$ independently of all the other vertices. At time $t \in \mathbb{N}$, the state of each node $i$ will be updated according to a deterministic process: if a node $i$ was active at time $t-1$, it will remains active at time $t$. Otherwise, $i$ will become active if at least $\theta\left(d_{i}\right)$ of its neighbors were active at time $t-1$. For some applications of this model we refer to [2], [18], [19], [22] and [26]. 
In the case where $\alpha(d)=\alpha$ and $\theta(d)=\theta$, for each $d \in \mathbb{N}$, our diffusion model is equivalent to what is called bootstrap percolation. This model has a rich history in statistical physics, mostly on $G=\mathbb{Z}^{d}$ and finite boxes. Bootstrap percolation was first mentioned and studied in the statistical physics literature by Chalupa et al. in [8]. The problem of complete occupation on $\mathbb{Z}^{2}$ was solved by van Enter in [25]. A short physics survey is [1]. Bootstrap percolation also has connections to the dynamics of the Ising model at zero temperature [11]. Bootstrap percolation on the random regular graph $G(n, d)$ with fixed vertex degree $d$ was studied by Balogh and Pittel [4]. Also Balogh et al. [3] studied bootstrap percolation on infinite trees.

Let $G$ be a graph with $n$ nodes, i.e., $|V|=n$. Let $A$ denote the adjacency matrix of $\mathrm{G}$, with $A_{i j}=1$ if $i \sim j$ and $A_{i j}=0$ otherwise. The state of the network at time $t$ can be described by the vector $\left(X_{i}(t)\right)_{i=1}^{n}: X_{i}(t)=1$ if the node $i$ is active at time $t$ and $X_{i}(t)=0$ otherwise. Remark that $X_{i}(0)$ is a Bernoulli random variable with parameter $\alpha\left(d_{i}\right)$. The evolution of this vector at time $t+1$ follows the following functional equation, i.e., at each time step $t+1$, each node $v$ applies:

$$
X_{i}(t+1)=X_{i}(t)+\left(1-X_{i}(t)\right) \mathbb{1}\left(\sum_{j} A_{i j} X_{j}(t) \geqslant \theta\left(d_{i}\right)\right) .
$$

From the definition, $X_{i}(t)$ is non-decreasing; sure-enough, the equation (1) implies again that $X_{i}(t+1) \geqslant X_{i}(t)$. Define $\Phi^{(n)}(\alpha, \theta, t)$ as

$$
\Phi^{(n)}(\alpha, \theta, t):=n^{-1} \sum_{j=1}^{n} \mathbb{E}\left[X_{j}(t)\right] .
$$

We are interested in finding the asymptotic value when $n \rightarrow \infty$, of

$$
\Phi^{(n)}(\alpha, \theta):=\lim _{t \rightarrow \infty} \Phi^{(n)}(\alpha, \theta, t)
$$

in the case of random graphs with given vertex degrees. The next section describes this model of random graphs.

\subsection{Random Graphs with Given node Degrees}

In this paper, we investigate random graphs with fixed given degree sequences (see for example Molloy and Reed [20, 21] and Janson [14]) as the underlying model for the interacting network, and analyze the above diffusion process on them. So ideally, we are interested in (uniformly chosen) random graphs having a prescribed degree sequence. But it is difficult to directly examine these random graphs, so instead, we use the configuration model (or ' $\mathrm{CM}$ ') which was introduced in this form by Bollobàs in [6] and motivated in part by the work of Bender and Canfield [5]. We briefly recall the definition of this model and refer to [6], [9] and [24] for more on this. 
For each integer $n \in \mathbb{N}$, we are given a sequence $D_{n}=\left(d_{n, i}\right)_{i=1}^{n}$ of nonnegative integers $d_{n, 1}, \ldots, d_{n, n}$ such that $\sum_{i=1}^{n} d_{n, i}$ is even. By $\mathbb{D}=\left\{D_{n}\right\}_{n}=\left\{\left(d_{n, i}\right)_{i=1}^{n}\right\}_{n}$ we done the family of all these given sequences. Define $\Omega_{D_{n}}$ to be the set of all (labeled) simple graphs with degree sequence $D_{n}$, i.e., the degree of the node $i$ is $d_{n, i}$. A random graph on $n$ vertices with degree sequence $D_{n}$ is a uniformly random member of $\Omega_{D_{n}}$ which we denote it by $G(n, \mathbb{D})$. Thus $G(n, \mathbb{D})$ is a random graph with degree sequence $D_{n}$ which has been uniformly chosen between all the graphs with $n$ nodes and having degree sequence $D_{n}$. We denote by $G(\mathbb{D})$ a random graph with degree sequence $\mathbb{D}$ which is a sequence of random graphs $G(n, \mathbb{D})$ where $n$ varies over integers.

A random multigraph with given degree sequence $D_{n}$, denoted by $C M(n, \mathbb{D})$, is defined by the following configuration model: Let $E_{i}$ denote a set of $d_{n, i}$ half-edges for each node $i$. (The sets $E_{i}$ are disjoint.) The half-edges are joined to form the set of edges of a multigraph on the set $\{1, \ldots, n\}$ in a very natural way: the set of all half-edges, i.e., the union $\cup E_{i}$, is partitioned into pairs and the two half-edges within a given pair are joined to form an edge. Each partition of the half-edges is called a configuration. The configuration is chosen uniformly at random over the set of all possible configurations. This procedure generates a graph with degree sequence $D_{n}$; however, the graph may contain loops and/or multiple edges. We denote by $C M(\mathbb{D})$ a random multigraph with degree sequence $\mathbb{D}$, i.e., a sequence of random multigraphs $C M(n, \mathbb{D})$. It is quite easy to see that, conditioned on the resulted multigraph being a simple graph, we obtain a uniformly distributed random graph with the given degree sequence $D_{n}$, which we have denoted by $G(n, \mathbb{D})$. The sequence $\mathbb{D}$ is assumed to satisfy the following regularity conditions (when $n \rightarrow \infty)$ :

Condition 1. For each $n, D_{n}=\left(d_{n, i}\right)_{i=1}^{n}$ is a sequence of non-negative integers such that $\sum_{i=1}^{n} d_{n, i}$ is even and, for some probability distribution $\left(p_{r}\right)_{r=0}^{\infty}$ over integers, independent of $n \in \mathbb{N}$, the following hold:

1. \#\{i: $\left.d_{n, i}=r\right\} / n \rightarrow p_{r}$ for every $r \geqslant 0$ as $n \rightarrow \infty$ (the degree density condition: the density of vertices of degree $r$ tends to $p_{r}$ );

2. $\lambda:=\sum_{r} r p_{r} \in(0, \infty)=\mathbb{E}_{p}(r)$ (finite expectation property);

3. $\sum_{i=1}^{n} d_{n, i} / n \rightarrow \lambda$ as $n \rightarrow \infty$ (the average degree tends to a given value $\lambda$ );

4. $\sum_{i=1}^{n} d_{n, i}^{2}=O(n)$ (second moment property).

When talking about a random graph with a given degree sequence $\mathbb{D}$, we consider the asymptotic case when $n \rightarrow \infty$ and say that an event holds w.h.p. (with high probability) if it holds with probability tending to one as $n$ goes to infinity. We shall use $\stackrel{p}{\rightarrow}$ for convergence in probability as $n \rightarrow \infty$. Similarly, we use $o_{p}$ and $O_{p}$ in a standard way. for example, if $\left(X_{n}\right)$ is a sequence of random variables, then $X_{n}=O_{p}(1)$ means that " $X_{n}$ is bounded in probability" and $X_{n}=o_{p}(n)$ means that $X_{n} / n \stackrel{p}{\rightarrow} 0$.

In the following we will need the following result of Janson. 
Theorem 2 (Janson [15]). Assume that $\mathbb{D}=\left\{D_{n}\right\}$ satisfies Condition 1. Then

$$
\liminf _{n \rightarrow \infty} \mathbb{P}(C M(n, \mathbb{D}) \text { is simple })>0 .
$$

As a corollary we obtain:

Corollary 3. Let $\mathbb{D}=\left\{D_{n}\right\}$ be a given fixed degree sequence satisfying Condition 1 . Then, an event $E_{n}$ occurs with high probability for $G(n, \mathbb{D})$ when it occurs with high probability for $C M(n, \mathbb{D})$.

Proof. Let $S_{n}$ be the event that $C M(n, \mathbb{D})$ is simple, $\mathbb{P}^{*}$ be the law of a uniform simple random graph $G(n, \mathbb{D})$, and $\mathbb{P}$ be the law of $C M(n, \mathbb{D})$. We recall that conditioned on the event $C M(n, \mathbb{D})$ being a simple graph, $C M(n, \mathbb{D})$ is a uniform simple random graph with that degree sequence. Hence

$$
\mathbb{P}^{*}\left(E_{n}\right)=\mathbb{P}\left(E_{n} \mid S_{n}\right)=1-\mathbb{P}\left(E_{n}^{c} \mid S_{n}\right)=1-\frac{\mathbb{P}\left(E_{n}^{c} \cap S_{n}\right)}{\mathbb{P}\left(S_{n}\right)} \geqslant 1-\frac{\mathbb{P}\left(E_{n}^{c}\right)}{\mathbb{P}\left(S_{n}\right)}
$$

By Theorem 2, $\liminf _{n \rightarrow \infty} \mathbb{P}\left(S_{n}\right)>0$. Moreover, $\lim _{n \rightarrow \infty} \mathbb{P}\left(E_{n}^{c}\right)=0$, then

$$
\lim _{n \rightarrow \infty} \frac{\mathbb{P}\left(E_{n}^{c}\right)}{\mathbb{P}\left(S_{n}\right)}=0
$$

This completes the proof.

Corollary 3 allows to prove a property for uniform graphs with a given degree sequence by proving it for the configuration model with that degree sequence.

\section{$1.2 \quad$ Main Results}

In this subsection, we state the main results of this work.

Let $D$ be a random variable with integer values and with distribution $\mathbb{P}(D=r)=p_{r}$, $r \in \mathbb{N}$. The two functions $\alpha: \mathbb{N} \rightarrow[0,1]$ and $\theta: \mathbb{N} \rightarrow \mathbb{N}$ are given as before. We define the function $f_{\alpha, \theta}:[0,1] \rightarrow \mathbb{R}$ as follows

$$
f_{\alpha, \theta}(y):=\lambda y^{2}-y \mathbb{E}[(1-\alpha(D)) D \mathbb{1}(\operatorname{Bin}(D-1,1-y)<\theta(D))] .
$$

Let $y^{*}=y_{\alpha, \theta}^{*}$ be the largest solution to $f_{\alpha, \theta}(y)=0$, i.e.,

$$
y^{*}:=\max \left\{y \in[0,1] \mid f_{\alpha, \theta}(y)=0\right\} .
$$

Remark that such $y^{*}$ exists because $y=0$ is a solution and $f_{\alpha, \theta}$ is continuous. The main result of this paper is the following theorem.

Theorem 4. Let $\mathbb{D}$ be a given degree sequence satisfying Condition 1 and let $G(n, \mathbb{D})$ be a (simple) random graph with degree sequence $\mathbb{D}$. Then we have: 
1. If $\theta(d) \leqslant d$ for all $d \in \mathbb{N}$ and furthermore $y^{*}=0$, i.e., if $f_{\alpha, \theta}(y)>0$ for all $y \in(0,1]$, then w.h.p. $\Phi^{(n)}(\alpha, \theta)=1-o_{p}(1)$.

2. If $y^{*}>0$ and furthermore $y^{*}$ is not a local minimum point of $f_{\alpha, \theta}(y)$, then w.h.p.

$$
\Phi^{(n)}(\alpha, \theta)=1-\mathbb{E}\left[(1-\alpha(D)) \mathbb{1}\left(\operatorname{Bin}\left(D, 1-y^{*}\right)<\theta(D)\right)\right]+o_{p}(1) .
$$

The second theorem of this paper is the following:

Theorem 5 (The cascade condition). Let $\mathbb{D}$ be a given degree sequence satisfying Condition 1 and let $G(n, \mathbb{D})$ be a (simple) random graph with degree sequence $\mathbb{D}$. There exists a single node $v$ which can trigger a global cascade, i.e., $v$ can activate a strictly positive fraction of the total population w.h.p. if and only if $\mathbb{E}[D]<\mathbb{E}\left[D(D-1) \mathbb{1}_{(\theta(D)=1)}\right]$.

Remark 6. We note that in the case where $\theta(d)=\theta d$, Watts [26] obtained the same condition by a heuristic argument validated through simulations. Our theorem provides as a very special case a mathematical proof of his heuristic results.

In the rest of this introductory section, we provide some of the applications of our main theorems above. But let us first briefly explain the methods used to derive Theorems 4 and 5. The base of our approach is some standard techniques similar to those used by Balogh and Pittel [4] for the special $d$-regular case problem, Cain and Wormald [7] for the $k$-core problem and Molloy and Reed [21] for the giant component problem. This means we consider the diffusion process on the random configuration model and describe the dynamics of the diffusion by a Markov chain. The proof of Theorem 4 is mainly based on a method introduced by Wormald in [27] for the analysis of a discrete random process by using differential equations. However, our model is more general and new difficulties arise in treating the Markov chain and proving the convergence results. One special difficulty is that, contrary to [4], here the number of variables is a function of $n$ (and so is not constant). We need also to generalize slightly Wormald's theorem to cover the case of an infinite number of variables. The proof of Theorem 5 is based on Theorem 4 and a theorem of Janson [14] for the study of percolation in a random graph with given vertex degrees. We refer to Section 3 for more details.

$k$-Core in Random Graphs with Given Degree Sequence. Let $k \geqslant 2$ be a fixed integer. The $k$-core of a given graph $G$, denoted by $\operatorname{Core}_{k}(G)$, is the largest induced subgraph of $G$ with minimum vertex degree at least $k$. The $k$-core of an arbitrary finite graph can be found by removing vertices of degree less than $k$, in an arbitrary order, until no such vertices exist. Let Core $_{k}^{(n)}$ be the expected number of vertices in the graph $\operatorname{Core}_{k}(G(n, \mathbb{D}))$.

The existence of a large $k$-core in a random graph with a given degree sequence has been studied by several authors, see for example Fernholz and Ramachandran [10] and Janson and Luczak [16]. Theorem 4 allows us to unify all these results into a single 
theorem. In fact by assuming the functions $\alpha$ and $\theta$ to be equal to $\hat{\alpha}(d)=\mathbb{1}(d<k)$ and $\hat{\theta}(d)=(d-k+1)_{+}=(d-k+1) \mathbb{1}(d \geqslant k)$ respectively, we obtain

$$
\frac{\operatorname{Core}_{k}^{(n)}}{n}=1-\Phi^{(n)}(\hat{\alpha}, \hat{\theta})
$$

Let $\hat{y}=y_{\hat{\alpha}, \hat{\theta}}^{*}$ be the largest solution to $f_{\hat{\alpha}, \hat{\theta}}(y)=0$.

Corollary 7 (Janson-Luczak [16]). Let $\mathbb{D}$ be a given degree sequence satisfying Condition 1 and let $G(n, \mathbb{D})$ be a (simple) random graph with degree sequence $\mathbb{D}$. Then we have:

1. If $\hat{y}=0$, i.e., if $f_{\hat{\alpha}, \hat{\theta}}(y)>0$ for all $y \in(0,1]$, then w.h.p. $\operatorname{Core}_{k}^{(n)}=o(n)$.

2. If $\hat{y}>0$ and furthermore $\hat{y}$ is not a local minimum point of $f_{\hat{\alpha}, \hat{\theta}}(y)$, then w.h.p. $\operatorname{Core}_{k}^{(n)}=n \mathbb{P}(\operatorname{Bin}(D, \hat{y}) \geqslant k) n+o(n)$.

Bootstrap Percolation on Random Regular Graphs. In the case of random regular graphs, i.e., in the case $d_{i}=d$ for all $i$, our diffusion model is equivalent to bootstrap percolation. Bootstrap percolation on the random regular graph $G(n, d)$ with fixed vertex degree $d$ was studied by Balogh and Pittel in [4]. By Theorem 4 we can recover a large part of their results. Let $A_{f}$ be the final set of active vertices. We find that

Corollary 8 (Balogh-Pittel [4]). Let the three parameters $\alpha, \theta$ and $d \in[0,1]$ be given with $1 \leqslant \theta \leqslant d-1$. Consider the bootstrap percolation on the random d-regular graph $G(n, d)$ in which each vertex is initially active independently at random with probability $\alpha$ and the threshold is $\theta$. Let $\alpha_{c}$ be defined as follows

$$
\alpha_{c}:=1-\inf _{0<y \leqslant 1} \frac{y}{\mathbb{P}(\operatorname{Bin}(d-1,1-y) \leqslant \theta-1)} .
$$

We have

(i) If $\alpha>\alpha_{c}$, then $\left|A_{f}\right|=n-o_{p}(n)$.

(ii) If $\alpha<\alpha_{c}$, then w.h.p. a positive proportion of the vertices remain inactive. More precisely, if $y^{*}=y^{*}(\alpha)$ is the largest $y \leqslant 1$ such that $\mathbb{P}(\operatorname{Bin}(d-1,1-y) \leqslant \theta-1) / y$ $=(1-\alpha)^{-1}$, then

$$
\frac{\left|A_{f}\right|}{n} \stackrel{p}{\rightarrow} 1-(1-\alpha) \mathbb{P}\left(\operatorname{Bin}\left(d, 1-y^{*}\right) \leqslant \theta-1\right)<1 .
$$

Proof. It remains only to show that in case $(i i), y^{*}$ is not a local minimum point of

$$
f_{\alpha, \theta}(y)=d y^{2}\left(1-(1-\alpha) \frac{\mathbb{P}(\operatorname{Bin}(d-1,1-y) \leqslant \theta-1)}{y}\right) .
$$

In fact, $\mathbb{P}(\operatorname{Bin}(d-1,1-y) \leqslant \theta-1) / y$ is decreasing when $\theta=d-1$ and has only one minimum point when $\theta<d-1$ (see [4] for details). Thus for $\theta<d-1$, the only local minimum point is the global minimum point $\hat{y}$ with $\mathbb{P}(\operatorname{Bin}(d-1,1-\hat{y}) \leqslant \theta-1) / \hat{y}=$ $\left(1-\alpha_{c}\right)^{-1}$ and otherwise, when $\theta=d-1$, there is no local minimum point. 
In this case, Balogh and Pittel [4] have also studied the threshold in greater detail by allowing $\alpha$ to depend on $n$; we have

- if $n^{1 / 2}\left(\alpha(n)-\alpha_{c}\right) \rightarrow \infty$, then w.h.p. $\left|A_{f}\right|=n$;

- if $n^{1 / 2}\left(\alpha_{c}-\alpha(n)\right) \rightarrow \infty$, then w.h.p. $\left|A_{f}\right|<n$ and furthermore

$$
\left|A_{f}\right|=n\left(1-(1-\alpha(n)) \mathbb{P}\left(\operatorname{Bin}\left(d, 1-y^{*}\right) \leqslant \theta-1\right)\right)+O_{p}\left(n^{1 / 2}\left(\alpha_{c}-\alpha(n)\right)^{-1 / 2}\right) .
$$

It would be interesting to generalize these results to our case. For this we need to obtain a quantitative version of Wormald's theorem for the case of an infinite number of variables. Quantitative version for the case of a finite number of variables has been recently obtained in [23]. Note that Balogh and Pittel [4] do not use Wormald's theorem. Indeed they analyze directly the system of differential equations via exponential supermartingales by using its integrals to show that the percolation process undergoes relatively small fluctuations around the deterministic trajectory.

\subsection{Organization of the Paper}

Diffusion process on $C M(n, \mathbb{D})$ is studied in detail in Section 2.1. The proof of our results are based on the use of differential equations for solving discrete random processes, and this is due to Wormald [27]. This is also discussed in Section 2.2. The proofs of our main results, Theorem 4 and Theorem 5, are given in Section 3.

\section{Diffusion Process in $C M(n, \mathbb{D})$}

In this section we provide the mathematical tools we need for the proof of our main theorems in Section 3.

\subsection{The Markov Chain}

The aim of this section is to describe the dynamics of the diffusion process as a Markov chain, which is perfectly tailored for the asymptotic study. We first describe the diffusion process on $C M(n, \mathbb{D})$ where the sequence $\mathbb{D}=\left\{D_{n}\right\}, D_{n}=\left(d_{n, i}\right)_{i=1}^{n}$, satisfies Condition 1. Let $2 m(n):=\sum_{i=1}^{n} d_{n, i}$ denote the number of half-edges in the configuration model.

Let us introduce the sets $S_{1}, \ldots, S_{n},\left|S_{i}\right|=d_{n, i}$, representing the vertices $1, \ldots, n$, respectively. Let $M_{n}$ be a uniform random matching on $S=\cup_{i} S_{i}$ which gives us $C M(n, \mathbb{D})$. Let $\mathbf{A}(0)$ and $\mathbf{I}(0)$ be the initial sets of active and inactive vertices, respectively. In particular we have $V=\mathbf{A}(0) \biguplus \mathbf{I}(0)$. Let $S_{i}(0):=S_{i}$ denote the initial set of half-edges hosted by the vertex $i$. We call the half-edges of a subset $S_{i}(t)$ active (resp. inactive) if $i \in \mathbf{A}(t)$ (resp. $i \in \mathbf{I}(t))$. We define the following process: in step 0, we pick a pair $(a, b)$, with $a \in S_{i}$ and $b \in S_{j}$ such that $i \in \mathbf{A}(0)$, and then delete both $a$ and $b$ from $S_{i}$ and 
$S_{j}$ respectively. Recursively, after $t$ steps, we have the set of (currently) active vertices at step $t, \mathbf{A}(t)$, and the set of (currently) inactive vertices at step $t, \mathbf{I}(t)$. We also denote by $S_{i}(t)$ the state of set $S_{i}$ at step $t$. At step $t+1$, we do the following

- We pick an active half-edge $a \in S_{i}(t)$ for $i \in \mathbf{A}(t)$;

- We identify its partner $b:(a, b) \in M_{n}$;

- And we delete both $a$ and $b$ from the sets $S_{i}(t)$ and $S_{j}(t)$;

- If $j$ is currently inactive, and $b$ is the $\theta\left(d_{j}\right)$-th half-edge deleted from the initial set $S_{j}$, then $j$ becomes active from this moment on.

The system is described in terms of

- $A(t)$ : the number of half-edges belonging to active vertices at time $t$;

- $I_{d, j}(t), 0 \leqslant j<\theta(d)$, the number of inactive nodes with degree $d$, and $j$ deleted half-edges, i.e., $j$ active neighbors at time $t$;

- $I(t)$ the number of inactive nodes at time $t$.

It is easy to see that the following identities hold:

$$
\begin{aligned}
A(t) & =\sum_{i \in \mathbf{A}(t)}\left|S_{i}(t)\right| . \\
I_{d, j}(t) & =\left|\left\{i \in \mathbf{I}(t): d_{i}=d,\left|S_{i}(t)\right|=d-j\right\}\right|, \quad 0 \leqslant j<\theta(d) . \\
I(t) & =\sum_{d} \sum_{j=0}^{\theta(d)-1} I_{d, j}(t) .
\end{aligned}
$$

Because at each step we delete two half-edges and the number of half-edges at time 0 is $2 m(n)$, the number of existing half-edges at time $t$ will be $2 m(n)-2 t$ and we have

$$
A(t)=2 m(n)-2 t-\sum_{d} \sum_{j<\theta(d)}(d-j) I_{d, j}(t) .
$$

The process will finish at the stopping time $T_{f}$ which is the first time $t \in \mathbb{N}$ where $A(t)=0$. The final number of active vertices will be $\left|A_{f}\right|=n-I\left(T_{f}\right)$. By the definition of our process $\left\{A(t),\left\{I_{d, j}(t)\right\}_{d, j<\theta(d)}\right\}_{t \geqslant 0}$ is Markov. We write the transition probabilities of the Markov chain. There are three possibilities for $B$, the partner of a half-edge $e$ of an active node $A$ at time $t+1$.

1. $B$ is active. The probability of this event is $\frac{A(t)}{2 m(n)-2 t-1}$, and we have

$$
\begin{aligned}
A(t+1) & =A(t)-2, \\
I_{d, j}(t+1) & =I_{d, j}(t), \quad(0 \leqslant j<\theta(d)) .
\end{aligned}
$$


2. $B$ is inactive of degree $d$ and the half-edge $e$ is the $(k+1)$-th deleted half-edge, and $k+1<\theta(d)$. The probability of this event is $\frac{(d-k) I_{d, k}(t)}{2 m(n)-2 t-1}$, and we have

$$
\begin{aligned}
& A(t+1)=A(t)-1, \\
& I_{d, k}(t+1)=I_{d, k}(t)-1, \\
& I_{d, k+1}(t+1)=I_{d, k+1}(t)+1, \\
& I_{d, j}(t+1)=I_{d, j}(t), \text { for } 0 \leqslant j<\theta(d), j \neq k, k+1 .
\end{aligned}
$$

3. $B$ is inactive of degree $d$ and $e$ is the $\theta(d)$-th deleted half-edge of $B$. The probability of this event is $\frac{(d-\theta(d)+1) I_{d, \theta(d)-1}}{2 m(n)-2 t-1}$. The next state is

$$
\begin{aligned}
& A(t+1)=A(t)+d-\theta(d)-1, \\
& I_{d, j}(t+1)=I_{d, j}(t), \quad(0 \leqslant j<\theta(d)-1), \\
& I_{d, \theta(d)-1}(t+1)=I_{d, \theta(d)-1}(t)-1 .
\end{aligned}
$$

Let $F_{t}$ denote the pairing generated by time $t$, i.e., $F_{t}=\left\{e_{1}, e_{2}\right\}$ be the set of halfedges picked at time $t$. We obtain the following equations for expectation of $A(t+1)$, $\left\{I_{d, j}(t+1)\right\}_{d, j<\theta(d)}$ conditioned on $A(t),\left\{I_{d, j}(t)\right\}_{d, j<\theta(d)}$ :

$$
\begin{aligned}
\mathbb{E}\left[A(t+1)-A(t) \mid F_{t}\right] & =-1+\frac{-A(t)+\sum_{d}(d-\theta(d)+1)(d-\theta(d)) I_{d, \theta(d)-1}(t)}{2 m-2 t-1}, \\
\mathbb{E}\left[I_{d, 0}(t+1)-I_{d, 0}(t) \mid F_{t}\right] & =-\frac{d I_{d, 0}(t)}{2 m-2 t-1}, \\
\mathbb{E}\left[I_{d, j}(t+1)-I_{d, j}(t) \mid F_{t}\right] & =\frac{(d-j+1) I_{d, j-1}(t)-(d-j) I_{d, j}(t)}{2 m-2 t-1} .
\end{aligned}
$$

\subsection{The Differential Equation Method}

In this section we briefly present a method introduced by Wormald in [27] for the analysis of a discrete random process by using differential equations. In particular we recall a general purpose theorem for the use of this method. This method has been used to analyze several kinds of algorithms on random graphs and random regular graphs (see for example [7], [21] and [28]).

Recall that a function $f\left(u_{1}, \ldots, u_{j}\right)$ satisfies a Lipschitz condition on $\Omega \subset \mathbb{R}^{j}$ if a constant $L>0$ exists with the property that

$$
\left|f\left(u_{1}, \ldots, u_{j}\right)-f\left(v_{1}, \ldots, v_{j}\right)\right| \leqslant L \max _{1 \leqslant i \leqslant j}\left|u_{i}-v_{i}\right|
$$

for all $\left(u_{1}, \ldots, u_{j}\right)$ and $\left(v_{1}, \ldots, v_{j}\right)$ in $\Omega$. For variables $I_{1}, \ldots, I_{b}$ and for $\Omega \subset \mathbb{R}^{b+1}$, the stopping time $T_{\Omega}\left(I_{1}, \ldots, I_{b}\right)$ is defined to be the minimum $t$ such that $\left(t / n ; I_{1}(t) / n, \ldots, I_{b}(t) / n\right) \notin$ $\Omega$. This is written as $T_{\Omega}$ when $I_{1}, \ldots, I_{b}$ are understood from the context. For simplicity the dependence on $n$ is usually dropped from the notation. 
The following theorem is a reformulation of Theorem 5.1 of [28], modified and extended for the case of an infinite number of variables. In it, "uniformly" refers to the convergence implicit in the $o()$ terms. Hypothesis (1) ensures that $I_{t}$ does not change too quickly throughout the process. Hypothesis (2) tells us what we expect for the rate of change to be, and property (3) ensures that this rate does not change too quickly. The proof of this theorem is given in the Appendix.

Theorem 9 (Wormald [28]). Let $b=b(n)$ be given (b is the number of variables). For $1 \leqslant l \leqslant b$, suppose $I_{l}(t)$ is a sequence of real-valued random variables such that $0 \leqslant$ $I_{l}(t) \leqslant C n$ for some constant $C$, and $F_{t}$ be the history of the sequence, i.e., the sequence $\left\{I_{j}(k), 0 \leqslant j \leqslant b, 0 \leqslant k \leqslant t\right\}$.

Suppose also that for some bounded connected open set $\Omega=\Omega(n) \subseteq \mathbb{R}^{b+1}$ containing the intersection of $\left\{\left(t, i_{1}, \ldots, i_{b}\right): t \geqslant 0\right\}$ with some neighborhood of the domain

$$
\left\{\left(0, i_{1}, \ldots, i_{b}\right): \mathbb{P}\left(I_{l}(0)=i_{l} n, 1 \leqslant l \leqslant b\right) \neq 0 \text { for some } n\right\},
$$

the following three conditions are verified:

1. (Boundedness). For some function $\beta=\beta(n) \geqslant 1$ and for all $t<T_{\Omega}$

$$
\max _{1 \leqslant l \leqslant b}\left|I_{l}(t+1)-I_{l}(t)\right| \leqslant \beta .
$$

2. (Trend). For some function $\lambda=\lambda_{1}(n)=o(1)$ and for all $l \leqslant b$ and $t<T_{\Omega}$,

$$
\left|\mathbb{E}\left[I_{l}(t+1)-I_{l}(t) \mid H_{t}\right]-f_{l}\left(t / n, I_{1}(t) / n, \ldots, I_{l}(t) / n\right)\right| \leqslant \lambda_{1} .
$$

3. (Lipschitz). For each $l$ the function $f_{l}$ is continuous and satisfies a Lipschitz condition on $\Omega$ with all Lipschitz constants uniformly bounded.

Then the following holds

(a) For $\left(0, \hat{i}_{1}, \ldots, \hat{i}_{b}\right) \in \Omega$, the system of differential equations

$$
\frac{d i_{l}}{d s}=f_{l}\left(s, i_{1}, \ldots, i_{l}\right), \quad l=1, \ldots, b,
$$

has a unique solution in $\Omega, i_{l}: \mathbb{R} \rightarrow \mathbb{R}$ for $l=1, \ldots, b$, which passes through $i_{l}(0)=\hat{i}_{l}, l=1, \ldots, b$, and which extends to points arbitrarily close to the boundary of $\Omega$.

(b) Let $\lambda>\lambda_{1}$ with $\lambda=o(1)$. For a sufficiently large constant $C$, with probability $1-O\left(\frac{b \beta}{\lambda} \exp \left(-\frac{n \lambda^{3}}{\beta^{3}}\right)\right)$, we have

$$
I_{l}(t)=n i_{l}(t / n)+O(\lambda n)
$$

uniformly for $0 \leqslant t \leqslant \sigma n$ and for each $l$. Here $i_{l}(t)$ is the solution in (a) with $\hat{i}_{l}=I_{l}(0) / n$, and $\sigma=\sigma(n)$ is the supremum of those $s$ to which the solution can be extended before reaching within $l^{\infty}$-distance $C \lambda$ of the boundary of $\Omega$. 
We note that $f_{l}$ depends only on $s$ and $i_{1}, \ldots, i_{l}$. This is to avoid complicated issues around the solutions of infinite sets of differential equations. We will also use the following corollary of the above theorem, which is namely Theorem 6.1 of [28]. This theorem states that, as long as condition 3 holds in $\Omega$, the solution of the system of equations above can be extended beyond the boundary of $\hat{\Omega}$, into $\Omega$.

Corollary 10 (Wormald [28]). For any set $\hat{\Omega}=\hat{\Omega}(n) \subseteq \mathbb{R}^{b+1}$, let $T_{\hat{\Omega}}=T_{\hat{\Omega}(n)}\left(I_{1}, \ldots, I_{b}\right)$ be the minimum $t$ such that $\left(\frac{t}{n}, \frac{I_{1}(t)}{n}, \ldots, \frac{I_{b}(t)}{n}\right) \notin \hat{\Omega}$ (the stopping time). Assume in addition that the first two hypotheses of Theorem 9 are verified but only within the restricted range $t<T_{\hat{\Omega}}$ of $t$. Then the conclusions of the theorem hold as before, after replacing $0 \leqslant t \leqslant \sigma n$ by $0 \leqslant t \leqslant \min \left\{\sigma n, T_{\hat{\Omega}}\right\}$.

Proof. For $1 \leqslant j \leqslant b$, define the random variables $\hat{I}_{j}$ by

$$
\hat{I}_{j}(t+1)= \begin{cases}I_{j}(t+1) & \text { if } t<T_{\hat{\Omega}}, \\ I_{j}(t)+f_{j}\left(t / n, I_{1}(t) / n, \ldots, I_{j}(t) / n\right) & \text { otherwise }\end{cases}
$$

for all $t \geqslant 0$. The $\hat{I}_{j}$ 's satisfy the hypotheses of Theorem 9 , and so the corollary follows since $\hat{I}_{j}(t)=I_{j}(t)$ for $0 \leqslant t<T_{\hat{\Omega}}$.

\section{Proofs of the Main Theorems}

In this section we present the proofs of Theorem 4 and Theorem 5 .

\subsection{Proof of Theorem 4}

The proof of Theorem 4 is mainly based on Theorem 9 . Indeed we will apply this theorem to show that the trajectory of $I_{d, j}$ throughout the algorithm is a.a.s. close to the solution of the deterministic differential equations suggested by these equations.

Let us define $\Delta_{n}:=\max \left\{d_{n, i}\right\}$ and let $b(n):=\sum_{d \leqslant \Delta_{n}} d . \theta(d)$. For simplicity the dependence on $n$ is dropped from the notations. For $\epsilon>0$, we define the domains $\Omega(\epsilon)$ as

$$
\begin{array}{r}
\Omega(\epsilon):=\left\{\left(\tau,\left\{i_{d, j}\right\}_{j<\theta(d)}\right) \in \mathbb{R}^{b(n)+1}:-\epsilon<i_{d, j}<\lambda,-\epsilon<\tau<\frac{\lambda\left(1-\epsilon^{2}\right)}{2},\right. \\
\left.\lambda-2 \tau-\sum_{d, j<\theta(d)}(d-j) i_{d, j}>0\right\} .
\end{array}
$$

Let $T_{\Omega}$ be the stopping time for $\Omega$ which is the first time $t$ when

$$
\left(t / n,\left\{I_{d, j}(t) / n\right\}\right) \notin \Omega .
$$

Let (DE) be the following system of differential equations:

$$
\begin{aligned}
& i_{d, 0}^{\prime}(\tau)=\frac{-d i_{d, 0}(\tau)}{\lambda-2 \tau}, \\
& i_{d, j}^{\prime}(\tau)=\frac{(d-j+1) i_{d, j-1}(\tau)-(d-j) i_{d, j}(\tau)}{\lambda-2 \tau} \quad(0<j<\theta(d)),
\end{aligned}
$$


with initial conditions

$$
i_{d, 0}(0)=p_{d}(1-\alpha(d)), \quad i_{d, j}(0)=0 \text { for } 0<j<\theta(d) .
$$

We have

Lemma 11. 1. The system (DE) has a unique solution in $\Omega(\epsilon)$ which extends to points arbitrarily close to the boundary of $\Omega(\epsilon)$.

2. For a sufficiently large constant $C$, with high probability we have

$$
I_{d, j}(t)=n i_{d, j}(t / n)+o(n) .
$$

uniformly for all $t \leqslant n \sigma$. Here $\sigma=\sigma(n)$ is the supremum of those $\tau$ for which the solution of these differential equations can be extended before reaching within $l^{\infty}$-distance $C n^{-1 / 4}$ of the boundary of $\Omega(\epsilon)$.

Proof. We will use Theorem 9. The domain $\Omega(\epsilon)$ is a bounded open set which contains all initial values of variables which may happen with positive probability. Each variable is bounded by a constant times $n$. By the definition of our process, the Boundedness Hypothesis is satisfied with $\beta(n)=1$. Trend Hypothesis is satisfied by some $\lambda_{1}(n)=$ $O(1 / n)$. Finally the third condition (Lipschitz Hypothesis) of the theorem is also satisfied since $\lambda-2 \tau$ is bounded away from zero. Then we set $\lambda=O\left(n^{-1 / 4}\right)>\lambda_{1}$. The conclusion of Theorem 9 now gives

$$
I_{d, j}(t)=n i_{d, j}(t / n)+O\left(n^{3 / 4}\right)
$$

with probability $1-O\left(n^{7 / 4} \exp \left(-n^{1 / 4}\right)\right)$ uniformly for all $t \leqslant n \sigma$. Finally, for $0<j<\theta(d)$, we have $I_{d, j}(0)=0$, and by Condition $1, I_{d, 0}(0) / n \stackrel{p}{\rightarrow} p_{d}(1-\alpha(d))$. This completes the proof.

To analyze $\sigma$, we need to determine which constraint is violated when the solution reaches the boundary of $\Omega(\epsilon)$. It cannot be the first constraint, because (5) must give asymptotically feasible values of $I_{d, j}$ until the boundary is approached. It remains to determine which of the last two constraints is violated when $\tau=\sigma$.

We first solve the system of differential equations (DE) and then we analyze the point up to which the resulting equations are valid. Note that these equations are similar to those used in the special $d$-regular case in [4].

Lemma 12. The solution of the system of differential equations (DE) is

$$
i_{d, j}(\tau)=p_{d}(1-\alpha(d))\left(\begin{array}{l}
d \\
j
\end{array}\right) y^{d-j}(1-y)^{j}
$$

where $y=(1-2 \tau / \lambda)^{1 / 2}$. 
Proof. Let $u=u(\tau)=-\frac{1}{2} \ln (\lambda-2 \tau)$. Then $u(0)=-\frac{1}{2} \ln (\lambda), u$ is strictly monotone and so is the inverse function $\tau=\tau(u)$. Let $f_{d, j}(u)=i_{d, j}(\tau(u))$. We write the system of differential equations above with respect to $u$ :

$$
\begin{aligned}
f_{d, 0}^{\prime}(u) & =-d f_{d, 0}(u) \\
f_{d, j}^{\prime}(u) & =(d-j+1) f_{d, j-1}(u)-(d-j) f_{d, j}(u) .
\end{aligned}
$$

Then using

$$
\frac{d}{d u}\left(f_{d, j}(u) e^{(d-j-1)(u-u(0))}\right)=e^{(d-j-1)(u-u(0))}(d-j) f_{d, j}(u)
$$

and by induction, we find

$$
f_{d, j}(u)=e^{-(d-j)(u-u(0))} \sum_{r=0}^{j}\left(\begin{array}{l}
d-r \\
j-r
\end{array}\right)\left(1-e^{-(u-u(0))}\right)^{j-r} f_{d, r}(u(0)),
$$

$0 \leqslant j \leqslant \theta(d)-1$. By going back to $\tau$, we have

$$
i_{d, j}(\tau)=y^{d-j} \sum_{r=0}^{j} i_{d, j}(0)\left(\begin{array}{l}
d-r \\
j-r
\end{array}\right)(1-y)^{j-r}, y=(1-2 \tau / \lambda)^{1 / 2} .
$$

It is then easy to finish the proof.

Let us define

$$
\begin{aligned}
a(\tau) & :=\lambda-2 \tau-\sum_{d, j<\theta(d)}(d-j) i_{d, j}(\tau), \quad \text { and } \\
i(\tau) & :=\sum_{d, j<\theta(d)} i_{d, j}(\tau) .
\end{aligned}
$$

Lemma 13. Assume $\sigma=\sigma(n)$ be the same as in Lemma 11. For $t \leqslant n \sigma$, we have

$$
|I(t) / n-i(t / n)| \stackrel{p}{\rightarrow} 0,
$$

and

$$
|A(t) / n-a(t / n)| \stackrel{p}{\rightarrow} 0 .
$$

Proof. By definition, we have

$$
\begin{aligned}
|I(t) / n-i(t / n)| & =\left|\sum_{d, j<\theta(d)}\left(I_{d, j}(t) / n-i_{d, j}(t / n)\right)\right| \\
& \leqslant \sum_{d, j<\theta(d)} d\left|I_{d, j}(t) / n-i_{d, j}(t / n)\right|, \\
|A(t) / n-a(t / n)| & =\left|2 m(n) / n-\lambda-\sum_{d, j<\theta(d)}(d-j)\left(I_{d, j}(t) / n-i_{d, j}(t / n)\right)\right| \\
& \leqslant|2 m(n) / n-\lambda|+\sum_{d, j<\theta(d)} d\left|I_{d, j}(t) / n-i_{d, j}(t / n)\right| .
\end{aligned}
$$


By Condition 1 , we have $|2 m(n) / n-\lambda| \stackrel{p}{\rightarrow} 0$. To complete the proof, it suffices to prove

$$
\sum_{d, j<\theta(d)} d\left|I_{d, j}(t) / n-i_{d, j}(t / n)\right| \stackrel{p}{\rightarrow} 0 .
$$

By Lemma 11, for each $d$ and $j<\theta(d)$, we have $\left|I_{d, j}(t) / n-i_{d, j}(t / n)\right| \stackrel{p}{\rightarrow} 0$. Hence, the same holds for any finite partial sum, which is for each $K \in \mathbb{N}$, we have

$$
\sum_{d<K, j<\theta(d)} d\left|I_{d, j}(t) / n-i_{d, j}(t / n)\right| \stackrel{p}{\rightarrow} 0 .
$$

Finally, let $\epsilon>0$. By Condition $1, \sum_{d} d p_{d} \rightarrow \lambda \in(0, \infty)$. Then, there exist a constant $K$, such that $\sum_{d \geqslant K} d p_{d}<\epsilon$. Let $N(d)$ denote the number of vertices with degree $d$ at time 0. By Lemma 12,

$$
i_{d, j}(\tau)=p_{d}(1-\alpha(d))\left(\begin{array}{l}
d \\
j
\end{array}\right) y^{d-j}(1-y)^{j} \leqslant p_{d}
$$

Again, by Condition 1,

$$
\sum_{d} d N(d) / n \longrightarrow \sum_{d} d p_{d}
$$

Also

$$
\sum_{d \geqslant K} d N(d) / n \longrightarrow \sum_{d \geqslant K} d p_{d}<\epsilon .
$$

Therefore, if $n$ is large enough, $\sum_{d \geqslant K} d N(d) / n<\epsilon$, and

$$
\begin{aligned}
\sum_{d \geqslant K, j<\theta(d)} d\left|I_{d, j}(t) / n-i_{d, j}(t / n)\right| & \leqslant \sum_{d \geqslant K, j<\theta(d)} d\left(I_{d, j}(t) / n+i_{d, j}(t / n)\right) \\
& \leqslant \sum_{d \geqslant K, j<\theta(d)} d\left(N(d) / n+p_{d}\right)<2 \epsilon .
\end{aligned}
$$

This completes the proof.

We now return to the proof of Theorem 4. By Lemma 12, we have

$$
\begin{aligned}
i(\tau) & =\sum_{d, j<\theta(d)} p_{d}(1-\alpha(d))\left(\begin{array}{l}
d \\
j
\end{array}\right) y^{d-j}(1-y)^{j} \\
& =\mathbb{E}[(1-\alpha(D)) \mathbb{1}(\operatorname{Bin}(D, 1-y)<\theta(D))], \quad \text { and } \\
a(\tau) & =\lambda-2 \tau-\sum_{d} \sum_{j<\theta(d)}(d-j) i_{j}^{(d)}(\tau) \\
& =\lambda y^{2}-y \mathbb{E}[(1-\alpha(D)) D \mathbb{1}(\operatorname{Bin}(D-1,1-y)<\theta(D))] \\
& =: f_{\alpha, \theta}(y),
\end{aligned}
$$


where $y=(1-2 \tau / \lambda)^{1 / 2}$ and $D$ is a random variable with distribution $\mathbb{P}(D=r)=p_{r}$. There are two cases:

First assume $f_{\alpha, \theta}(y)>0$ for all $y \in(0,1]$, i.e., $y^{*}=0$. Then we have

$$
\lambda-2 \tau-\sum_{d, j<\theta(d)}(d-j) i_{d, j}>0
$$

in $\Omega(\epsilon)$. So the boundary reached is determined by $\hat{\tau}=\frac{\lambda\left(1-\epsilon^{2}\right)}{2}$ which is $y=\epsilon$. Then by $\theta(d)<d, i(\hat{\tau})=O(\epsilon)$ and Lemma 13 implies $\left|A_{f}\right|=n-O(n \epsilon)$. This proves the first part of the theorem.

Now consider the case $y^{*}>0$, and further $y^{*}$ is not a local minimum point of $f_{\alpha, \theta}(y)$. Then by definition of $y^{*}$ and by using the fact that $f_{\alpha, \theta}(1) \geqslant 0$, we have $f_{\alpha, \theta}(y)<0$ for some interval $\left(y^{*}-\epsilon, y^{*}\right)$. Then the first constraint is violated at time $\hat{\tau}=\frac{1-\lambda y^{* 2}}{2}$. We apply Corollary 10 with $\hat{\Omega}$ the domain $\Omega(\epsilon)$ defined above, and the domain $\Omega$ replaced by $\Omega^{\prime}(\epsilon)$, which is the same as $\Omega$ except that the constraint $a>0$ is omitted:

$$
\Omega^{\prime}(\epsilon)=\left\{\left(\tau,\left\{i_{d, j}\right\}_{j<\theta(d)}\right) \in \mathbb{R}^{b(n)+1}:-\epsilon<i_{d, j}<\lambda, \quad-\epsilon<\tau<\frac{\lambda\left(1-\epsilon^{2}\right)}{2}\right\} .
$$

This gives us the convergence in equations (5) upto the point where the solution leaves $\Omega^{\prime}(\epsilon)$ or when $A(t)>0$ is violated. Since $a(\tau)$ begins to go negative after $\hat{\tau}$, from equation (9) it follows that $A(t)>0$ must be violated a.a.s., and it becomes zero at some $T_{f} \sim \hat{\tau} n$. Hence by equation (8), we conclude

$$
\left|A_{f}\right|=n-I\left(T_{f}\right)=n-n \mathbb{E}\left[(1-\alpha(D)) \mathbb{1}\left(\operatorname{Bin}\left(D, 1-y^{*}\right)<\theta(D)\right)\right]+o_{p}(n),
$$

which completes the proof for $C M(n, \mathbb{D})$. Now it suffices to use Corollary 3 to transfer the result from $C M\left(n, D_{n}\right)$ to $G\left(n, D_{n}\right)$.

\subsection{Proof of Theorem 5}

For each node $i$, let $C_{i}$ denote the final set of active nodes when in the starting state of the procedure the node $i$ is the only active node. Clearly if $j \in C_{i}$, then $C_{j} \subseteq C_{i}$. Let $\alpha(d)=\alpha$ for each $d \in \mathbb{N}$. We define $\gamma_{\theta}(D):=\frac{\mathbb{E}[D(D-1) \mathbf{1}(\theta(D)=1)]}{\mathbb{E}[D]}$.

We first prove that if $\gamma_{\theta}(D)>1$, then there exists a single node which can activate a positive fraction of the population. To do this, we use a theorem of Janson [14] about the existence of a giant component in the percolated graph. (The term giant component is used for the existence of a component in a graph containing at least a fraction $c$ of all vertices for some positive constant $c>0$ which does not depend on $n$. The question of existence of a giant component in $G(n, \mathbb{D})$ was answered by Molloy and Reed [21], who showed that a giant component exists w.h.p. if and only if (in the notation above) $\mathbb{E}[D(D-2)]>0$.) 
Given any graph $G$ and a probability $\pi: \mathbb{N} \rightarrow[0,1]$, we denote by $G_{\pi}$ the random graph obtained by randomly deleting every vertex $v \in G$ with probability $1-\pi\left(d_{v}\right)$. Thus for each node $v, \pi\left(d_{v}\right)$ denotes the probability to be kept in the percolation model. Fountoulakis [12] and Janson[14] show that for this percolation model on $G(n, \mathbb{D})$, if we condition the resulting random graph on its degree sequence $\tilde{\mathbb{D}}$, and let $\tilde{n}$ be the number of its vertices, then the graph has the distribution of $G(\tilde{n}, \tilde{\mathbb{D}})$, the random graph with this degree sequence. They calculate then the distributions of the degree sequence $\tilde{\mathbb{D}}$ and finally apply known results to $G(\tilde{n}, \tilde{\mathbb{D}})$.

To conclude the proof of our Theorem 5, we will use the following theorem.

Theorem 14 (Janson [14]). Let $\mathbb{D}$ be a given degree sequence satisfying Condition 1 and let $G(n, \mathbb{D})$ be a (simple) random graph with degree sequence $\mathbb{D}$. Consider the site percolation model $G(n, \mathbb{D})_{\pi}$. Suppose further that there exists $d \geqslant 1$ such that $p_{d}>0$ and $\pi(d)<1$. Then w.h.p. there is a giant component if and only if

$$
\sum_{d=0}^{\infty} d(d-1) \pi(d) p_{d}>\lambda .
$$

Now consider $\pi_{\theta}(d)=\mathbb{1}(\theta(d)=1)$. Thus $G(n, \mathbb{D})_{\pi_{\theta}}$ is the random graph obtained by deleting all the nodes of $G(n, \mathbb{D})$ for which the threshold is greater than 1 . Hence $G(n, \mathbb{D})_{\pi_{\theta}}$ is a subgraph of $G(n, \mathbb{D})$ and we have

$$
v \in G(n, \mathbb{D})_{\pi_{\theta}} \text { if and only if } v \in G(n, \mathbb{D}) \& \theta\left(d_{v}\right)=1 .
$$

It is clear that to prove the existence of a node $v$ which can trigger a global cascade in $G(n, \mathbb{D})$ w.h.p., it suffices to prove that w.h.p. there is a giant component in the random percolated graph $G(n, \mathbb{D})_{\pi_{\theta}}$. Indeed the threshold of every node in the giant component of $G(n, \mathbb{D})_{\pi_{\theta}}$ is equal to one and then each node in the giant component can activate the whole component. By Theorem 14, there is w.h.p. a giant component in $G(n, \mathbb{D})_{\pi_{\theta}}$ if and only if

$$
\begin{aligned}
\lambda & <\sum_{d=0}^{\infty} d(d-1) \pi_{\theta}(d) p_{d} \\
& =\sum_{d=0}^{\infty} d(d-1) \mathbb{1}(\theta(d)=1) p_{d} \\
& =\mathbb{E}[D(D-1) \mathbb{1}(\theta(D)=1)] .
\end{aligned}
$$

We now prove that if $\gamma_{\theta}(D)<1$, then a single active node cannot activate a positive fraction of the population. We will actually prove that if $\gamma_{\theta}(D)<1$, w.h.p. we will have $\lim _{\alpha \rightarrow 0} \Phi(\alpha, \theta)=0$ which implies the claim. Define

$$
\begin{aligned}
f_{\theta}(y) & :=\lim _{\alpha \rightarrow 0} f_{\alpha, \theta}(y) \\
& =\lambda y^{2}-y \mathbb{E}[D \mathbb{1}(\operatorname{Bin}(D-1,1-y)<\theta(D))] .
\end{aligned}
$$


Clearly we have $f_{\theta}(1) \geqslant 0$. We claim that if $\gamma_{\theta}(D)<1$, then $f_{\theta}(1-\epsilon)<0$ for sufficiently small $\epsilon>0$. Indeed, we have

$$
\begin{aligned}
f_{\theta}(1-\epsilon) & =\lambda(1-\epsilon)^{2}-(1-\epsilon) \mathbb{E}[D \mathbb{1}(\operatorname{Bin}(D-1, \epsilon)<\theta(D))] \\
& =\lambda(1-\epsilon)^{2}-(1-\epsilon)(\lambda-\mathbb{E}[D \mathbb{1}(\operatorname{Bin}(D-1, \epsilon) \geqslant \theta(D))]) \\
& =\lambda(1-2 \epsilon)-(1-\epsilon)(\lambda-\mathbb{E}[D(D-1) \mathbb{1}(\theta(D)=1)] \epsilon)+o(\epsilon) \\
& =(-\lambda+\mathbb{E}[D(D-1) \mathbb{1}(\theta(D)=1)]) \epsilon+o(\epsilon),
\end{aligned}
$$

which is negative for $\gamma_{\theta}(D)<1$. We infer that w.h.p. $\lim _{\alpha \rightarrow 0} y^{*}=1$. And this in turn implies, by Theorem 4, that w.h.p. $\lim _{\alpha \rightarrow 0} \Phi(\alpha, \theta)=0$. This completes the proof.

\section{Conclusion and Future Work}

We have studied diffusion and bootstrap percolation in a random graph with a given degree sequence. Our main result is a theorem which enables to find the final proportion of the active vertices in the asymptotic case, i.e., when $n \rightarrow \infty$. It would be interesting to obtain quantitative versions of our results, such as large deviation estimates and central limit theorems. But this seems to be more involved due to the generality of our model. (See for example [17] for some related work on the particular problem of $k$-core). These and some other related issues are left to a future work.

Acknowledgements. I would like to thank an anonymous referee for the suggestions which improved the presentation of the paper. Special thanks to Omid Amini, François Baccelli, Moez Draief and Marc Lelarge for helpful comments and discussions.

\section{References}

[1] J. Adler and U. Lev. Bootstrap percolation: visualizations and applications. Brazilian Journal of Physics, 33(3):641-644, 2003.

[2] H. Amini, M. Draief, and M. Lelarge. Marketing in a random network. In Proceedings of NetCoop08, LNCS 5425, pages 17-25, 2009.

[3] J. Balogh, Y. Peres, and G. Pete. Bootstrap percolation on infinite trees and nonamenable groups. Combinatorics, Probability and Computing, 15(5):715-730, 2006.

[4] J. Balogh and B. G. Pittel. Bootstrap percolation on the random regular graph. Random Structures \&3 Algorithms, 30(1-2):257-286, 2007.

[5] E. A. Bender and E. R. Canfield. The asymptotic number of labeled graphs with given degree sequences. Journal of Combinatorial Theory, Ser. A, 24:296-307, 1978.

[6] B. Bollobás. Random Graphs. Cambridge University Press, 2001.

[7] J. Cain and N. Wormald. Encores on cores. Electronic Journal of Combinatorics 13 (2006), \#R81.

[8] J. Chalupa, G. R. Reich, and P. L. Leath. Bootstrap percolation on a bethe lattice. Journal of Physics C, 12:L31-L35, 1979. 
[9] R. Durrett. Random graph dynamics. Cambridge University Press, Cambridge, 2007.

[10] D. Fernholz and V. Ramachandran. Cores and connectivity in sparse random graphs. Technical Report TR-04-13, The University of Texas at Austin, Department of Computer Sciences, 2004.

[11] L. R. Fontes, R. H. Schonmann, and V. Sidoravicius. Stretched exponential fixation in stochastic ising models at zero temperature. Communications in Mathematical Physics, 228:495-518, 2002.

[12] N. Fountoulakis. Percolation on sparse random graphs with given degree sequence. Internet Mathematics, 4(4):329-356, 2007.

[13] W. Hurewicz. Lectures on Ordinary Differential Equations. M.I.T. Press, 1958.

[14] S. Janson. On percolation in random graphs with given vertex degrees. Electronic Journal of Probability, 14:86-118, 2009.

[15] S. Janson. The probability that a random multigraph is simple. Combinatorics, Probability and Computing, 18(1-2):205-225, 2009.

[16] S. Janson and M. Luczak. A simple solution to the k-core problem. Random Structures \& Algorithms, 30:50-62, 2007.

[17] S. Janson and M. J. Luczak. Asymptotic normality of the $k$-core in random graphs. Annals of Applied Probability, 18:1085, 2008.

[18] J. Kleinberg. Cascading behavior in networks: Algorithmic and economic issues. In Algorithmic Game Theory. Cambridge University Press, 2007.

[19] M. Lelarge. Diffusion of innovations on random networks: Understanding the chasm. In Proceedings of WINE 2008, pages 178-185, 2008.

[20] M. Molloy and B. Reed. A critical point for random graphs with a given degree sequence. Random Structures \&6 Algorithms, 6:161-179, 1995.

[21] M. Molloy and B. Reed. The size of the giant component of a random graph with a given degree sequence. Combinatorics, Probability and Computing, 7:295-305, 1998.

[22] S. Morris. Contagion. Review of Economic Studies, 67(1):57-78, 2000.

[23] T. G. Seierstad. A central limit theorem via differential equations. Annals of Applied Probability, 19:661, 2009.

[24] R. van der Hofstad. Random Graphs and Complex Networks, http://www.win.tue.nl/ rhofstad/NotesRGCN.pdf. 2009.

[25] A. C. D. van Enter. Proof of straley's argument for bootstrap percolation. Journal of Statistical Physics, 48(3-4):943-945, 1987.

[26] D. J. Watts. A simple model of global cascades on random networks. Proceedings of the National Academy of Sciences, 99(9):5766-5771, 2002.

[27] N. Wormald. Differential equations for random processes and random graphs. Annals of Applied Probability, 5(4):1217-1235, 1995.

[28] N. Wormald. The differential equation method for random graph processes and greedy algorithms. In Lectures on Approximation and Randomized Algorithms, 1999. 


\section{Appendix}

Proof of Theorem 9. The solution is unique from a standard result in the theory of first order differential equations (see Hurewicz[13], Chapter 2, Theorem 11). We now present the proof of part (b). We will use the following supermartingale inequality. The proof follows from exactly the same proof as Azuma's inequality (see [28], Lemma 4.2).

Lemma 15. Let $\left\{X_{i}\right\}_{i=0}^{t}$ be a supermartingale with $X_{0}=0$ and $X_{i}-X_{i-1} \leqslant c_{i}$ for $i \geqslant 1$ and some constants $c_{i}$. Then for all $\alpha>0$,

$$
\mathbb{P}\left(X_{t} \geqslant \alpha\right) \leqslant \exp \left(-\frac{\alpha^{2}}{2 \sum c_{i}^{2}}\right) .
$$

Let us define $\omega=\lceil n \lambda / \beta\rceil, \alpha=n \lambda^{3} / \beta^{3}$ and let $0 \leqslant t \leqslant \sigma n$. If $\omega<n^{2 / 3}$ then $\beta / \lambda>n^{1 / 3}$ and then the probability in the conclusion is not restricted and there is nothing to prove.

Lemma 16. For some constant $B$ with probability $1-O\left(e^{-\alpha}\right)$, we have

$$
\left|I_{l}(t+\omega)-I_{l}(t)-\omega f_{l}\left(t / n, I_{1}(t) / n, \ldots, I_{l}(t) / n\right)\right|<B \omega \lambda .
$$

Proof. For $0 \leqslant k<\omega$, we have $k \beta / n=O(\lambda)$ and by the Trend and Lipschitz hypotheses

$$
\begin{aligned}
\mathbb{E}\left[I_{l}(t+k+1)-I_{l}(t+k) \mid H_{t+k}\right] & =f_{l}\left(\frac{t+k}{n}, \frac{I_{1}(t+k)}{n}, \ldots, \frac{I_{l}(t+k)}{n}\right)+O(\lambda) \\
& =f_{l}\left(t / n, I_{1}(t) / n, \ldots, I_{l}(t) / n\right)+O(\lambda) .
\end{aligned}
$$

Hence there exists a function $g(n)=O(\lambda)$ such that conditional on $F_{t}$,

$$
f(k):=I_{l}(t+k)-I_{l}(t)-k f_{l}\left(t / n, I_{1}(t) / n, \ldots, I_{l}(t) / n\right)-k g(n)
$$

is a supermartingale with respect to the sequence $\sigma$-fields generated by $F_{t}, \ldots, F_{t+\omega}$. By the boundedness hypothesis

$$
|f(k+1)-f(k)| \leqslant \beta+O(1) \leqslant \kappa \beta
$$

for some constant $\kappa>0$. Therefore, by Lemma 15,

$$
\mathbb{P}\left(\left|I_{l}(t+\omega)-I_{l}(t)-\omega f_{l}\left(t / n, I_{1}(t) / n, \ldots, I_{l}(t) / n\right)\right| \geqslant \omega g(n)+\kappa \beta \sqrt{\omega \alpha} \mid F_{t}\right) \leqslant 2 e^{-\alpha},
$$

and so the lemma follows.

Let $i=\lfloor n \sigma / \omega\rfloor$, and let $h_{l}(k)=\left|I_{l}(k \omega)-n i_{l}(k \omega / n)\right|$ for $0 \leqslant k \leqslant i$. We have

$$
h_{l}(k+1) \leqslant h_{l}(k)+\left|A_{1}\right|+\left|A_{2}\right|+\left|A_{3}\right|
$$

where

$$
\begin{aligned}
& A_{1}=I_{l}((k+1) \omega)-I_{l}(k \omega)-\omega f_{l}\left(k \omega / n, I_{1}(k \omega) / n, \ldots, I_{l}(k \omega) / n\right), \\
& A_{2}=\omega i_{l}^{\prime}(k \omega / n)+i_{l}(k \omega / n) n-i_{l}((k+1) \omega / n) n, \\
& A_{3}=\omega f_{l}\left(k \omega / n, I_{1}(k \omega) / n, \ldots, I_{l}(k \omega) / n\right)-\omega i_{l}^{\prime}(k \omega / n) .
\end{aligned}
$$


By Lemma 16, we have for a suitable universal constant $B^{\prime},\left|A_{1}\right|<B^{\prime} \omega \lambda$ with probability $1-O\left(e^{-\alpha}\right)$ (This is the point where the assumption, the scaled variables not approaching within distance $C \lambda$ of the boundary of $\Omega$, is justified). Since $f_{l}$ satisfies the Lipschitz hypothesis, we have

$$
\left|A_{2}\right|=O\left(n\left(\frac{\omega}{n}\right)^{2}\right)<B^{\prime \prime} \omega^{2} / n
$$

for a suitable constant $B^{\prime \prime}$. Finally using the same arguments as above we obtain

$$
\left|A_{3}\right|<\frac{B^{\prime \prime} \omega}{n} h_{l}(k) .
$$

Set $B=\max \left\{B^{\prime}, B^{\prime \prime}\right\}$. By induction on $k$, we infer that

$$
\mathbb{P}\left(h_{l}(k) \geqslant B_{k} \text { for some } k \leqslant i, 1 \leqslant l \leqslant b\right)=O\left(b i e^{-\alpha}\right),
$$

where

$$
B_{k}=B \omega(\lambda+\omega / n)\left((1+B \omega / n)^{k}-1\right) \frac{n}{B \omega} .
$$

We have $B_{k}=O(n \lambda+\omega)=O(n \lambda)$ since $\beta$ is bounded below. This proves the theorem in the case $t=k \omega$. Assume $t \leqslant n \sigma$. From time $\lfloor t / \omega\rfloor \omega$ to $t$ the change in $I$ and $i$ is at most $\omega \beta=O(n \lambda)$ and the theorem follows. 